\title{
Application of STATCOM for Damping Subsynchronous Resonance in a Multi-machine System
}

\author{
Yanlong Sun, Liangeng Ban, Daye Yang, Yizhen Wang \\ China Electric Power Research Institute, Beijing, China \\ Email: sunyanlong@epri.sgcc.com.cn
}

\begin{abstract}
Results of an investigation on the application of STATCOM for damping subsynchronous resonance (SSR) in a multi-machine system is presented in this paper. For a multi-machine system which has a set of identical parallel turbine-generators or non-identical turbine-generators having torsional modes of the same frequency, generators may suffer from the same mode of torsional interaction corresponding to a certain series compensation degrees. Generators in such system may have different oscillation behaviors when they are unequally loaded or have different shaft and electrical parameters. Serving as the grid-side equipment, the reactive power output of a STATCOM could have an impact on all generators electrical distance nearby. Thus a single STATCOM could be used to damp torsional interactions of multi-generators when additional proper control strategy is supplemented. In this paper, control strategy of using STATCOM to damp SSR in a multi-machine system is designed and its effectiveness is validated based on a modified IEEE second benchmark model.
\end{abstract}

Keywords: subsynchronous resonance (SSR); multi-machine system; STATCOM; mode partition controller (MPC).

\section{Introduction}

Areas that have an uneven distribution of energy and load need transmission of bulk power over long distance to realize the optimal arrangement of resources. For such long distance transmission systems, steady state stability limit is often the bottleneck factor that constrains the transmission capability of the system. Use of series capacitor compensation could reduce the equivalent reactance of the transmission line and significantly improve steady state stability limit of the system. Thus, series compensation has been widely used worldwide as a cost-effective measure to improve the transmission capability. However, transmission systems with series compensation may have an electromechanical coupling with the nearby turbine-generators, causing subsynchronous resonance (SSR) and cause loss to the fatigue life of the generator shaft.

For transmission systems with series compensation, its sending end generally has more than one unit, often with multiple units of a power plant or several power plants having short electrical distance from each other. Thus a system of multi-machine delivered through series compensation line is formed. For systems that have parallel identical turbine-generators or non-identical units having torsional modes of the same frequency, generators may suffer from the same mode of torsional interaction at a certain series compensation degrees. Reference [2, 3] conducted a research on the SSR characteristics of two kinds of multi-machine system. Results show that oscillation behaviors of unequally-loaded parallel identical generators and non-identical units having torsional modes of the same frequency may have different amplitude and phase, relating to their output level or modal parameter differences.

As a new type of reactive power compensation FACTS device, STATCOM has a fast and smooth control performance. By applying appropriate control strategies, STATCOM could be used to damp SSR. Taking generator speed as the input signal, [4] propose a way to excite additional electromagnetic torque at the modal frequency by injecting controllable compensation current into the generator using STATCOM, thus to damp torsional interaction of the generator. Reference [5] take local voltage as input signal and damp SSR by modulating the reactive current reference of STATCOM. By monitoring subsynchronous frequency current component in the transmission line and injecting corresponding compensation current into the grid using STATCOM, these current components could be prevented from flowing into the generator set, so as to achieve the intention of damping SSR [6].

Various control strategies have been designed to damp SSR using STATCOM in above references, but all these researches are based on the single-machine system, 
making these control strategies applicable only for single-machine system and parallel identical generators with same operating conditions [7]. For multi-machine system in which generators have different oscillation behavior, a STATCOM control strategy is proposed in this paper to damp torsional oscillations of all generators. All generators' speed is needed as the input signal to the STATCOM and a reactive power reference is generated through modal partition controller. Time domain simulations based on a modified IEEE second benchmark model are carried out to validate the effectiveness of the proposed strategy.

\section{System Modeling}

In multi machine system, torsional interaction occurs only between those units that have torsional modes of the same frequency. EPRI suggests that if corresponding torsional vibration frequencies of the units are different from each other more than $1 \%$, the original multi-machine system could be simplified into a single-machine system by reserving the unit concerned, while replacing other units by its subtransient reactance and a fixed voltage source. In addition, parallel identical generators with same operating conditions can be studied as an equivalent single unit. Therefore, in the study of the multi-machine system SSR, only unequally-loaded parallel identical generators and non-identical units having torsional modes of the same frequency are considered in this paper.

System II of the IEEE second benchmark model represents a system in which two non-identical generators that both have a torsional mode of $24.65 \mathrm{~Hz}$ deliver power through a series compensated line [8]. In order to incorporate unequally-loaded parallel identical generators into the model, an additional generator with the same parameters as the original 700MVA unit in [8] is added to the high-voltage bus in the power plant. Fig. 1 shows the one-line diagram of the modified IEEE second benchmark model, with STATCOM connected at the high-voltage bus in the power plant.

In fig.1, gen 1 has a capacity of 600MVA and its mechanical system comprises four masses, i.e., the high-pressure turbine (HP), the low-pressure turbine (LP), the generator (GEN), and the exciter (EXC), with three subsynchronous torsional oscillation modes of $24.65 \mathrm{~Hz} 、 32.39 \mathrm{~Hz}$ and $51.1 \mathrm{~Hz}$; gen 2 has a capacity of 700MVA and its mechanical system comprises three masses, i.e., the high-pressure turbine (HP), the low-pressure turbine (LP), and the generator (GEN), with two subsynchronous torsional oscillation modes of $24.65 \mathrm{~Hz}$ and $44.99 \mathrm{~Hz}$.

By adjusting gen 2 and gen 3 to different output level, this model comprises both unequally-loaded parallel identical generators and non-identical units having torsional modes of the same frequency. Thus it could represent various kinds of multi-machine system that we concern about. The STATCOM is adjusted to work at constant reactive power control.

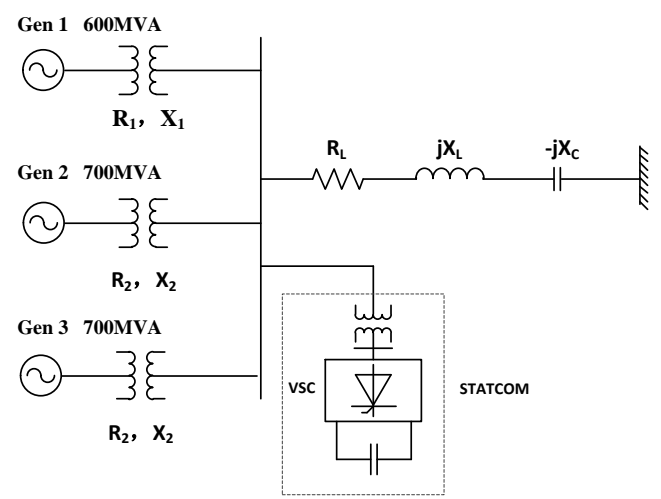

Fig. 1 Modified IEEE second benchmark model with STATCOM

\section{STATCOM Controller Design}

As mentioned in part 1, STATCOM could be applied to damp SSR of a single-machine system under a variety of control strategies, but not all these strategies can adapt to the damp of SSR in multi-machine system. Output of the generators will have a corresponding change when voltage of its export bus is modulated through the control of the reactive power output of the STATCOM, thus a controllable additional electromagnetic torque is induced on the generator shaft. With proper phase adjustment, this additional torque could be used to damp the torsional oscillation of the generator shaft. Since generators in most multi-machine system connect to a common sending bus, voltage modulation of the common bus using STATCOM could have an impact on all the generators. If the STATCOM is controlled based on the synthetic consideration of the different oscillation behaviors of all generators, SSR in multi-machine system could be damped using STATCOM installed on the common bus.

\subsection{Modal partition controller}

In order to damp SSR, STATCOM's reactive power output should use the generator speed as a reference. For single generator suffered from SSR, when its shaft speed rises, the STATCOM should be controlled to improve the bus voltage. Consequently, output of the generator as well as the electromagnetic torque on the shaft would increase. Under the condition of constant mechanical torque input on the shaft, STATCOM's 
regulation would play an decelerate effect on the generator. For condition that shaft speed decreases, the above process would be the opposite. Since STATCOM could regulate its reactive power output rapidly, it could be used to damp SSR.

Generator shaft usually has more than one torsional vibration mode. In order to avoid reciprocal influence between different mode control loops, structure of mode partition shown in Fig. 2 is used.

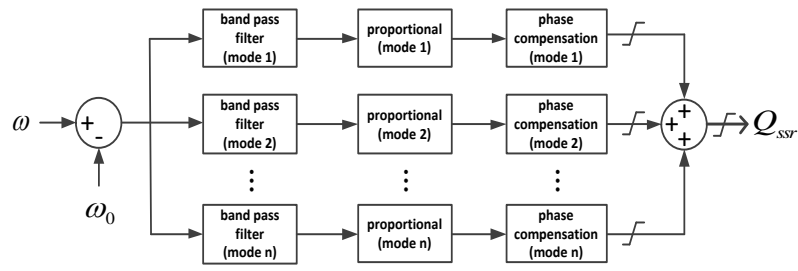

Fig. 2 Mode partition controller

The input signal of the mode partition controller (MPC) is the generator speed $\omega$. Speed deviation could be obtained by differencing $\omega$ and the synchronous speed $\omega 0$. Speed signal of each torsional mode could be separated from the speed deviation using specially designed high quality factor band-pass filter, which has a same center frequency as the corresponding torsional mode. Parameters of proportional and phase compensation component for each torsional mode could be designed independently due to the use of a parallel multi-channel controller structure. Summation of the output of each channel yields the reactive power reference $Q_{S S R}$ for the STATCOM to damp SSR. In order to avoid unexpected overregulation, limitation components are used at the end of each channel and the final output of the controller.

\subsection{Phase compensation component design}

The most critical part in the MPC design process is the determination of the phase compensation parameter for each mode channel. With inappropriate phase compensation, STATCOM may stimulate SSR rather than damp it. According to the complex torque coefficient method [9, 10], STATCOM can damp SSR when the phase difference between the additional electromagnetic torque caused by the control of STATCOM and the modal speed of the generator is in the range of $-90^{\circ}+90^{\circ}$.

Fig. 3 shows the overall flow diagram of damping SSR using STATCOM. Process from the additional reactive power reference $\mathrm{Q}_{\text {ref_ssR }}$ to the additional electromagnetic torque $\Delta \mathrm{T}_{\mathrm{e} \_\mathrm{SSR}}$ may cause some phase shift, depending on the phase characteristics of STATCOM and transmission system. In order to damp SSR, some phase com- pensation components are needed in the MPC to adjust the phase difference between $\Delta \mathrm{T}$ e_ssR and $\Delta \omega_{\mathrm{m}}$ into the range of $-90^{\circ} \sim+90^{\circ}$. With other components stay the same, maximum damping can be get whom $\mathrm{m}$ is in phase with $\Delta \mathrm{T}_{\mathrm{e} \_\mathrm{SSR}}$.

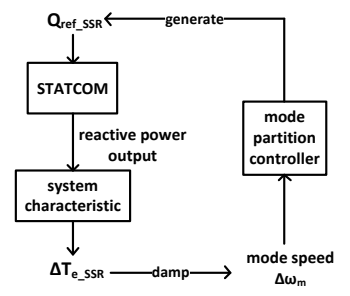

Fig. 3 overall flow diagram of damping SSR using STATCOM

Specific frequency signal can be used to calculate the phase difference needed to be compensated by MPC. When applying a sinusoidal reactive power instruction Qtest at a torsional frequency at STATCOM, corresponding additional electro-magnetic torque will emerge on the shaft. The corresponding frequency electromagnetic torque component $\Delta \mathrm{Te}$ _test can be s eparated using Fourier analysis. Phase difference $\Delta \varphi$ sys between Qtest and $\Delta$ Te test is just the total phase deviation of STATCOM and the transmission system at this particular frequency, thus MPC needs to compensate a phase in the range of $-\Delta \varphi \operatorname{sys} \pm 90^{\circ}$ in this mode channel.

\subsection{STATCOM Controller for Multi-machine System}

In the control strategy described above, output of STATCOM will affect the torsional frequency of the shaft slightly besides its damp of the torsional oscillation when phase difference betweenTe _SSR and $\Delta \omega \mathrm{m}$ is not $0^{\circ}$. If only one generator speed is taken as the input signal in a multi-machine system, only this generator's torsional oscillation could be damped. Although the initial phase difference between its mode speed and additional electromagnetic torque is in the range of $-90^{\circ} \sim$ $+90^{\circ}$, torsional oscillation still couldn't be damped due to the influence of STATCOM on the torsional frequency of its shaft for other generators. This phenomena has been verified by [2] based on a dual-machine system with SVC.

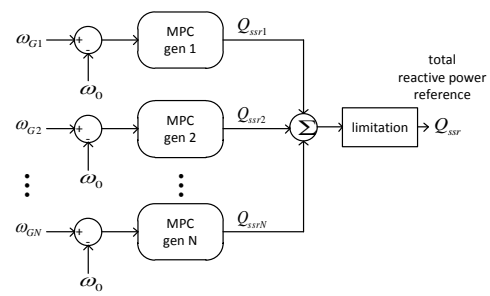

Fig. 4 STATCOM controller for Multi-machine system 
Different oscillation behaviors of multiple generators must be taken into consideration in the design of control strategy for damping SSR in a multi-machine system. By designing independent MPC for each generator and taking all generators' speed as input signal, a total reactive power reference can be synthesized for STATCOM to damp torsional oscillations of all generators.

\section{Digital Simulation}

To demonstrate the effectiveness of proposed control strategy for damping SSR in multi-machine system, the EMTDC/PSCAD program is used to do a simulation on the dynamic performance of the system modeled in section II. The analysis is carried based on the following initial operating condition and assumptions.
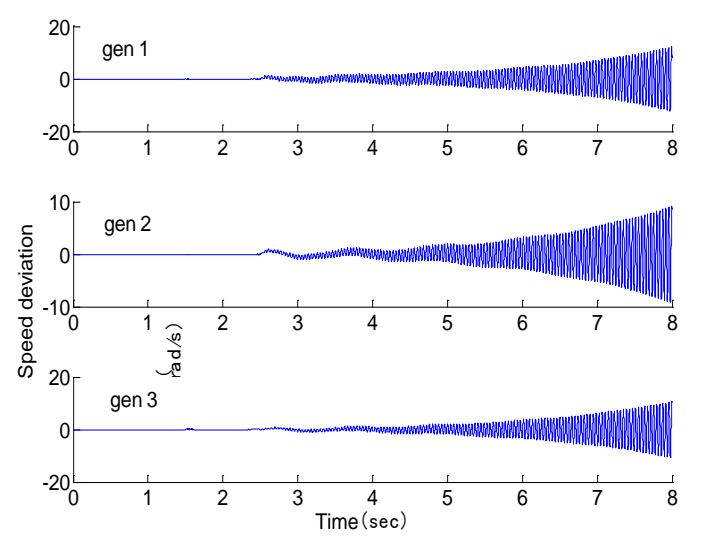

Fig. 5 System responses without SSR damping controller
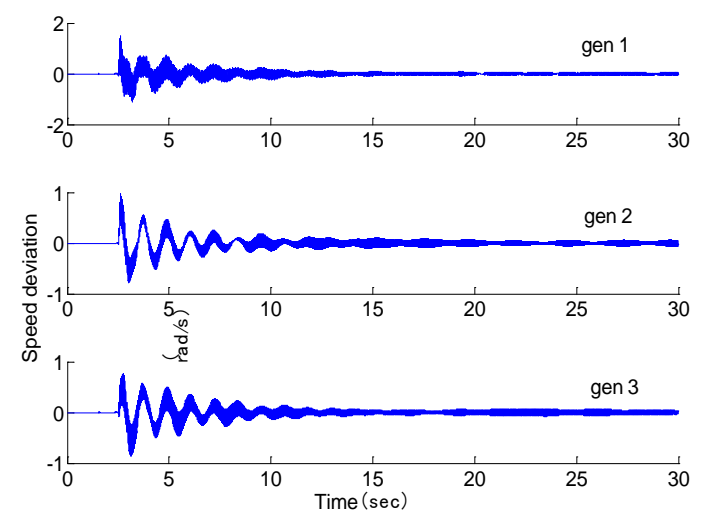

Fig. 6 System responses with SSR damping controller

1. Gen1, gen2, and gen3 deliver 0.9 p.u., 0.9 p.u., and 0.1 p.u. power to the transmission line respectively.
2. The input mechanical power to the three generators' turbine is constant.

3. The compensation level provided by the series capacitor is $47 \%$ of the line reactance XL.

4. A three phase line-to-ground instantaneous fault is applied at the infinite bus at $2.5 \mathrm{sec}$ and removed $0.01 \mathrm{sec}$ later.

At a compensation level of $47 \%$, the electric system has a negative damping at the common torsional frequency of the three generators. Without SSR damping control, the system is unstable after the fault is cleared. Speed deviation curve of the three unit obtained by transient simulation is shown in Fig. 5. It can also be seen from Fig. 5 that although the three units are oscillating at the same frequency, their amplitude are different as a result of different shaft parameter and output level.

Fig. 6 shows the simulation result of the speed deviation curve of the three units when multi-machine SSR damping controller is added to STATCOM. It can be seen from Fig. 6 that damping of the system has been greatly enhanced. The oscillations of all the three units' shaft decay with time, indicating the effectiveness of the multi-machine SSR damping controller.

\section{Conclusion}

This paper proposed a control strategy of using STATCOM to damp SSR in multi-machine system. The strategy is verified by time domain simulation of a multi-machine system modified based on IEEE second benchmark model. Dynamic response of the three generators' speed deviation without and with the SSR damping controller is presented. The simulation result reveals that by taking all generators' speed as input signal and with proper phase compensation, SSR in a multi-machine system can be damped using STATCOM.

\section{REFERENCES}

[1] Zheng Chao, Tang Xiaojun, Ma Shiying. “Analysis on Subsynchronous Oscillation damping characteristics of a SVC Light,” Electric Power, Vol. 43, No. 8, 2010, pp. 34-39.

[2] M.R.Iravani. "Torsional Oscillations of Unequally-loaded Parallel Identical Turbine-generators," IEEE Trans on Power Systems, Vol. 4, No. 4, 1989, pp. 1514-1524.

[3] G.D.Jennings, R.G.Harley. "Torsional Interaction Between Non-identical Turbine Generators,” IEEE Trans on Power Systems, Vol. 5, No. 1, 1990, pp. 133-139.

[4] Li Zhipeng, Xie Xiaorong. "STATCOM-SSR Damping Control Method Based on Dynamic Compensation of currents at Complementary Frequencies to Torsional Modes,” Proceedings of the CSEE, Vol. 30, No. 34, 2010, 
pp. 22-27.

[5] K.R.Padiyar, Nagesh Prabhu. "Design and Performance evaluation of Subsynchronous Damping Controller with STATCOM,” IEEE Trans on Power Delivery, Vol. 21, No. 3, 2006, pp. 1398-1405.

[6] Nagesh Prabhu, M.Janaki, R.Thirumalaivasan. "Damping of Subsynchronous Resonance by Subsynchronous Current Injector with STATCOM,” TENCON 2009 - 2009 IEEE Region 10 Conference, Singapore, 23-26 Jan. 2009, pp. 1-6.

[7] Jennings G.D., Grad S.A., Harley R.G., et al. "Subsynchronous resonance of a power station with two identical generating units,” IEEE Proceeding, Vol. 133, No. 1, 1986, pp. 33-43.

[8] IEEE Subsynchronous Resonance Working Group. "Second benchmark model for computer simulation of Subsynchronous resonance," IEEE Transactions on Power Apparatus and Systems, Vol. 104, No. 5, 1985, pp. 1057-1066.

[9] Canay I M.A. "Novel Approach to the torsional interaction and electrical damping of the synchronous machine: Part one-theory,” IEEE Transactions on Power Apparatus and Systems, Vol. 101, No. 10, 1982, pp. 3630-3638.

[10] Canay I M.A. "Novel Approach to the torsional interaction and electrical damping of the synchronous machine: Part two-application to an arbitrary Network," IEEE Transactions on Power Apparatus and Systems, Vol. 101, No. 10, 1982, pp. 3639-3646.
[11] Xu Zheng. "The complex torque coefficient approach's applicability analysis and its realization by time domain simulation,” Proceedings of the CSEE, Vol. 20, No. 6, 2000, pp. 1-4.

[12] K.V. Patil, J. Senthil, J. Jiang and R.M. Mathur. “Application of STATCOM for damping torsional oscillations in series compensated AC systems," IEEE Transactions on Energy Conversion, Vol. 13, No. 3, 1998, pp. 237-243.

[13] B.K. Keshavan, Nagesh Prabhu. "Damping of Subsynchronous Oscillations Using STATCOM-A FACTS Controller," International Conference on Power System Technology, Singapore, 21-24 Nov. 2004, pp. 12-16.

[14] Zhou Changchun, Xu Zheng, Wang Guan. "A Survey on Torsional Interaction in Multi-machine Power Grid," Power System Technology, Vol. 28, No. 6, 2004, pp.1-4.

[15] Zhang Fan, Xu Zheng. "Theory and Experiences of SVC for Damping Subsynchronous Resonance,” High Voltage Engineering, Vol. 33, No. 3, 2007, pp. 26-31.

[16] Cheng Shijie, Cao Yijia, Jiang Quanyuan. "Theory and method of power system subsynchronous oscillation," Power Beijing: Science Press, Beijing, 2008 , pp.68-96.

[17] Liu Shiyu, Xie Xiaorong, Wang Zhonghong. "SSR Problem in Compensated Transmission System of Thermal Power Bases in China,” Power System Technology, Vol. 32, No. 1, 2008, pp. 5-8. 